\title{
SELECTIONS OF SET-VALUED STOCHASTIC PROCESSES
}

\author{
MARIUSZ MICHTA and LONGIN E. RYBIŃSKI ${ }^{1}$ \\ Technical University, Institute of Mathematics \\ Podgórna 50, 65-246 Zielona Góra, Poland
}

(Received May, 1996; Revised January, 1997)

We show that $\mathscr{F}_{t}$-adapted, set-valued stochastic processes satisfying mild continuity conditions admit, $\mathcal{F}_{t}$-adapted, stochastically continuous selections.

Key words: Set-valued Stochastic Process, Conditional Expectation, Martingale, Measurable and Continuous Selections.

AMS subject classifications: $60 \mathrm{G} 20,60 \mathrm{G} 44,54 \mathrm{C} 60,28 \mathrm{~B} 20$.

\section{Introduction}

In this paper we prove several theorems on the existence of $\mathscr{F}_{t}$-adapted, continuous selections for $\mathcal{F}_{t}$-adapted, set-valued stochastic processes, as well as a continuous time version of Hess' result on martingale selection [3]. Such results may be useful in the theory of the set-valued stochastic integral.

\section{Preliminaries}

Let $(\Omega, \mathcal{F}, P)$ be a probability space with a filtration $\left(\mathcal{F}_{t}\right)_{t \geq 0}$ (i.e., with a family of $\sigma$ fields $\mathscr{F}_{t}$ ), such that $0 \leq s \leq t$ implies that $\mathscr{F}_{s} \subseteq \mathcal{F}_{t} \subseteq \mathcal{F}_{\text {. }}$. We assume that all $P$-null sets are in $\mathcal{F}_{0}$. Let $\mathscr{F}_{t-}=\sigma\left(\bigcup_{s \geq t} \mathscr{F}_{s}\right)$ and $\mathcal{F}_{t+}=\bigcap_{s>t} \mathcal{F}_{s}$. Obviously, $\mathscr{F}_{t-\subseteq}$ $\mathcal{F}_{t} \subseteq \mathcal{F}_{t+}$

For a random variable $\varphi: \Omega \rightarrow R^{n}$ such that $E(|\varphi|)=\int_{\Omega}|\varphi| d P<+\infty$, by $E\left(\varphi \mid \mathcal{F}_{t}\right)$ we denote the conditional expectation of $\varphi$, (i.e., an $\mathcal{F}_{t}$-measurable mapping) such that

for each $A \in \mathcal{F}_{t}$.

$$
\int_{A} E\left(\varphi \mid \mathcal{F}_{t}\right) d P=\int_{A} \varphi d P
$$

We say that a set-valued mapping $\Phi: \Omega \rightarrow R^{n}$ is a set-valued random variable iff $\Phi$

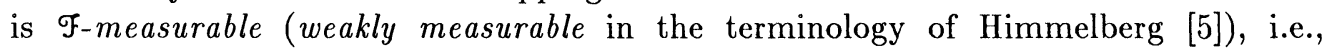

\footnotetext{
${ }^{1}$ This work has been supported by KBN Grant Number 332069203.
} 
$\{\omega: \Phi(\omega) \cap U \neq \emptyset\} \in \mathcal{F}$ for each open set $U \subseteq R^{n}$. Equivalently, $\Phi$ is $\mathcal{F}$-measurable iff the real-valued function $d(z, \Phi): \Omega \rightarrow R^{n}$ defined by

$$
d(z, \Phi)(\omega)=d(z, \Phi(\omega))=\inf _{v \in \Phi(\omega)}\|z-v\|,
$$

where $\|w\|$ is the Euclidean norm of $w \in R^{n}$, is a random variable. Clearly, for a mapping $\varphi: \Omega \rightarrow R^{n}$ identified with the set-valued mapping $\Phi=\{\varphi\}$, this is equivalent to saying that $\varphi$ is a random variable. Let $\left(F_{t}\right)=\left(F_{t}\right)_{t} \geq 0$ be a set-valued stochastic process with closed values in $R^{n}$ (i.e., a family of $\mathscr{F}$-measurable set-valued mappings $F_{t}: \Omega \rightarrow R^{n}, t \geq 0$, with closed values). We say that $\left(F_{t}\right)$ is $\mathcal{F}_{t^{-}}$adapted iff $F_{t}$ is $\mathcal{F}_{t^{-}}$ measurable for each $t \geq 0$, and we denote an $\mathcal{F}_{t}$-adapted process $\left(F_{t}\right)$ such that $E\left(d\left(0, F_{t}\right)\right)<+\infty$ for each $t \geq 0$, by $\left(F_{t}, \mathcal{F}_{t}\right)$. A selection of the process $\left(F_{t}\right)$ is a single-valued stochastic process $\left(f_{t}\right)$ such that for every $t \geq 0$, there holds $f_{t}(\omega) \in F_{t}(\omega)$ for $P$-almost all $\omega$. Additionally, if $\left(f_{t}\right)$ is $\mathscr{F}_{t}$-adapted and satisfies $E\left(\left|f_{t}\right|\right)<+\infty$ for each $t \geq 0$, we will denote the process by $\left(f_{t}, \mathscr{F}_{t}\right)$.

Let us mention that for the unique $\sigma$-field $\mathscr{F}$, the result on convergence of measurable selections being extracted from the sequence of measurable set-valued mappings, that converge in the distribution, has been investigated by Salinetti and Wets [9, Theorem 5.1, Corollary 5.2]. On the other hand, Hess has proven the existence of martingale selections for discrete time, set-valued martingales and discussed the convergence of set-valued martingales.

\section{Selection Theorem Results}

Our first simple result concerns the case when almost all paths $t \mapsto F_{t}(\omega)$ are continuous, and similar to the results of Salinetti and Wets, are based on the regularity of metric projections. For $z \in R^{n}$ and the closed, convex set $A \subset R^{n}$, we denote by $\operatorname{Pr}(z, A)$ the metric projection of $z$ onto $A$ with respect to Euclidean norm (i.e., a unique element $\mathrm{P} \mathrm{r}(z, A) \in A$ such that $\|\operatorname{Pr}(z, A)-z\|=d(z, A))$. The Wijsman topo$\log y$ for the family $C C l\left(R^{n}\right)$ of all nonempty, closed convex subsets of $R^{n}$, is the weakest topology such that for every $y \in R^{n}$, the function $A \mapsto d(y, A)$ is continuous [10]. We will need the following lemma.

Lemma 1: The mapping $A \mapsto \operatorname{Pr}(z, A)$ of $C C l\left(R^{n}\right)$ into $R^{n}$ is continuous with respect to the Wijsman topology.

Proof: For $A, A_{0} \in C C l\left(R^{n}\right)$ and $z \in R^{n}$, let us denote $y_{0}=\operatorname{Pr}\left(y_{0}, A\right)$, $y=\operatorname{Pr}(z, A)$. Clearly,

$$
\left\|y-y_{0}\right\| \leq\left\|y-\operatorname{Pr}\left(y_{0}, A\right)\right\|+\left\|\operatorname{Pr}\left(y_{0}, A\right)-y_{0}\right\|=\left\|y-\operatorname{Pr}\left(y_{0}, A\right)\right\|+d\left(y_{0}, A\right) .
$$

By the parallelogram equality, we have

$$
\begin{gathered}
\left\|y-\operatorname{Pr}\left(y_{0}, A\right)\right\|^{2}=2\|y-z\|^{2}+2\left\|\operatorname{Pr}\left(y_{0}, A\right)-z\right\|^{2}-4\left\|\frac{y+\operatorname{Pr}\left(y_{0}, A\right)}{2}-z\right\|^{2} \\
\leq 2\left\|\operatorname{Pr}\left(y_{0}, A\right)-z\right\|^{2}-2 d(z, A)^{2} .
\end{gathered}
$$

But 


$$
\left\|\operatorname{Pr}\left(y_{0}, A\right)-z\right\| \leq\left\|\operatorname{Pr}\left(y_{0}, A\right)-y_{0}\right\|+\left\|y_{0}-z\right\|=d\left(y_{0}, A\right)+d\left(z, A_{0}\right)
$$

Thus,

$$
\begin{gathered}
\left\|y-\operatorname{Pr}\left(y_{0}, A\right)\right\|^{2} \\
\leq 2\left(d\left(y_{0}, A\right)+d\left(z, A_{0}\right)-d(z, A)\right)\left(d\left(y_{0}, A\right)+d\left(z, A_{0}\right)+d(z, A)\right) .
\end{gathered}
$$

Consequently,

$$
\begin{gathered}
\left\|y-y_{0}\right\| \leq d\left(y_{0}<A\right) \\
+\sqrt{2} \sqrt{\left(d\left(y_{0}, A\right)+d\left(z, A_{0}\right)-d(z, A)\right)\left(d\left(y_{0}, A\right)+d\left(z, A_{0}\right)+d(z, A)\right)} .
\end{gathered}
$$

From this it follows immediately that $A \mapsto \operatorname{Pr}(z, A)$ is continuous with respect to the Wijsman topology.

Theorem 1: If the stochastic process $\left(F_{t}, \mathcal{F}_{t}\right)$ has closed convex values and for every $z \in R^{n}$, the functions $t \mapsto d\left(z, F_{t}\right)(\omega)$ is continuous for a.e. $\omega \in \Omega$, then for any $y \in R^{n}$, the process $\left(f_{t}\right)$ defined by $f_{t}(\omega)=\operatorname{Pr}\left(y, F_{t}(\omega)\right)$ is an $\mathcal{F}_{t^{-}}$-adapted selection of $F$ such that $t \mapsto f_{t}(\omega)$ is continuous for P-a.e. $\omega \in \Omega$.

Proof: By virtue of Lemma 1, from the assumption that the functions $t \mapsto d\left(z, F_{t}(\omega)\right), z \in R^{n}$, and a.e. $\omega \in \Omega$ are continuous, it follows that for every $y \in R^{n}$, a.e. $\omega \in \Omega$, the mapping $t \mapsto \operatorname{Pr}\left(y, F_{t}(\omega)\right)$ is continuous. To see that $f_{t}$ is $\mathcal{F}_{t}$-measurable note that

$$
\operatorname{Graph} f_{t}=\left\{(\omega, z):\|y-z\|-d\left(y, F_{t}(\omega)\right)=0\right\} \cap \operatorname{Graph} F_{t}
$$

Hence, by virtue of [5, Theorem 3.5 and Corollary 6.3], $f_{t}$ is $\mathscr{F}_{t}$-measurable.

In the following theorems we dispense completely with the upper semicontinuity assumption for the process $\left(F_{t}, \mathcal{F}_{t}\right)$. We do not adopt any lower semicontinuity assumption for the functions $t \mapsto d\left(y, F_{t}\right)(\omega)$; we assume only the stochastic upper semicontinuity of these functions, which means the stochastic lower semicontinuity of the process $\left(F_{t}, \mathcal{F}_{t}\right)$. We utilize a well-known theorem on measurable selections due to Kuratowski and Ryll-Nardzewski, as well as theorems on continuous selections of lower semicontinuous, set-valued mappings due to Michael [7] and to Antosiewicz, Cellina (see e.g., [1, Theorem 3]), respectively. We will need the following lemma.

Lemma 2: Assume that for the stochastic process $\left(F_{t}, \mathcal{F}_{t}\right), s \geq 0$ and every $z \in R^{n}, A \in \mathcal{F}_{s}$, the real-valued function $t \mapsto E\left(\chi_{A} d\left(z, F_{t}\right)\right)$ is right-hand (respectively: left-hand) usc at $s$. Then for any $\mathscr{F}_{s}$-measurable random variable $\varphi$ with $E(|\varphi|)<+\infty$, the function $t \mapsto E\left(d\left(\varphi, F_{t}\right)\right)$ is right-hand (respectively: left-hand) usc at $s$.

Proof: Let $\epsilon>0$. By assuming that for any constant function, $\varphi \equiv z$, we have $E\left(d\left(\varphi, F_{t}\right)\right)<E\left(d\left(\varphi, F_{s}\right)\right)+\frac{\epsilon}{2}$ whenever $t \in[s, s+\delta)$ (respectively, $t \in(s-\delta, s]$ ) for sufficiently small $\delta$. For a step random variable $\varphi=\sum_{i=1}^{m} z_{i} \chi_{A_{i}}, A_{i} \in \mathscr{F}_{s}$, we have

$$
E\left(d\left(\varphi, F_{t}\right)\right)=\sum_{i=1}^{m} E\left(\chi_{A_{i}} d\left(z_{i}, F_{t}\right)\right) \leq \sum_{i=1}^{m}\left(E\left(\chi_{A_{i}} d\left(z_{i}, F_{s}\right)\right)+\frac{\epsilon}{2^{i}}\right) \leq E\left(d\left(\varphi, F_{s}\right)\right)+\epsilon
$$

whenever $t \in[s, s+\delta)(t \in(s-\delta, s])$ for sufficiently small $\delta$. For an arbitrary $\mathcal{F}_{s}$ measurable $\varphi$, first choose a sequence of $\mathcal{F}_{s}$-measurable step functions $\varphi_{n}$ such that 
$E\left(\left|\varphi-\varphi_{n}\right|\right) \rightarrow 0$. Then choose $n$ such that $E\left(\left|\varphi-\varphi_{n}\right|\right)<\frac{\epsilon}{3}$ and let $\delta>0$ be such that $E\left(d\left(\varphi_{n}, F_{t}\right)\right)<E\left(d\left(\varphi_{n}, F_{s}\right)\right)+\frac{\epsilon}{3}$ for $t \in[s, s+\delta)(t \in(s-\delta, s])$. Then,

$$
\begin{gathered}
E\left(d\left(\varphi, F_{t}\right)\right) \leq E\left(\left|\varphi-\varphi_{n}\right|\right)+E\left(d\left(\varphi_{n}, F_{t}\right)\right)<E\left(d\left(\varphi_{n}, F_{s}\right)\right)+\frac{2}{3} \epsilon \\
\leq E\left(\left|\varphi_{n}-\varphi\right|\right)+E\left(d\left(\varphi, F_{s}\right)\right)+\frac{2}{3} \epsilon<E\left(d\left(\varphi, F_{s}\right)\right)+\epsilon,
\end{gathered}
$$

whenever $t \in[s, s+\delta)(t \in s-\delta, s])$.

Theorem 2: Assume that a set-valued stochastic process $\left(F_{t}, \mathcal{F}_{t}\right)$ has closed convex values and for every $z \in R^{n}, s \geq 0$, and $A \in \mathcal{F}_{s}$, the real-valued function $t \mapsto E\left(\chi_{A} d\left(z, F_{t}\right)\right)$ is right-hand usc at $s$. Then $\left(F_{t}, \mathcal{F}_{t}\right)$ has a $L^{1}$-right-hand continuous selection $\left(f_{t}, \mathscr{F}_{t}\right)$.

Proof: Define a set-valued mapping $G:[0,+\infty) \rightarrow L^{1}\left(\Omega, \mathcal{F}, R^{n}\right)$ by

$$
G(t)=\left\{\varphi \in L^{1}\left(\Omega, \mathcal{F}, R^{n}\right): \varphi \text { is } \mathcal{F}_{t} \text {-measurable selection of } F_{t}\right\}
$$

Based on the assumption $E\left(d\left(z, F_{t}\right)\right)<+\infty$ for each $t \geq 0$, the mapping $G$ has nonempty values by virtue of the Kuratowski and Ryll-Nardzewski measurable selection theorem (see e.g., [5, Theorem 5.1]). Moreover, the sets $G(t)$ are closed and convex because the set-valued random variables $F_{t}$ have closed, convex values. If we equip $[0,+\infty)$ with the arrow topology $\tau_{\rightarrow}$ (i.e., the topology generated by the intervals $[s, t), 0 \leq s<t)$, then it follows from the assumptions that $G:[0,+\infty) \rightarrow L\left(\Omega, \mathscr{F}, R^{n}\right)$ is a lower semicontinuous, set-valued mapping. Indeed, it suffices to show that $d(\varphi, G(t))=\inf _{\psi \in G(t)} E(|\varphi-\psi|) \rightarrow 0$ as $t \downarrow s$ for any $\varphi \in G(s), s \geq 0$. Since $\varphi$ is $F_{t^{-}}$ measurable for $t \geq s$, as a consequence of Kuratowski and Ryll-Nardzewski selection theorem, we have that

$$
d(\varphi, G(t))=E\left(d\left(\varphi, F_{t}\right)\right)
$$

for $t \geq s$, (see Hiai and Umegaki [4, Theorem 2.2] and Rybiński [8, Lemma 6]). But by virtue of Lemma 2 we have that $E\left(d\left(\varphi, F_{t}\right)\right) \rightarrow 0$ as $t \downarrow s$. This shows that $G$ is lower semicontinuous on $\left([0,+\infty), \tau_{\rightarrow}\right)$. Since $\left([0,+\infty), \tau_{\rightarrow}\right)$ is a Lindelöff space, hence paracompact (see Engelking [2]), we can then apply the Michael continuous selection theorem to $G$ ([7, Theorem $\left.\left.3.2^{\prime \prime}\right]\right)$, and get a continuous mapping $g:[0,+\infty) \rightarrow L^{1}\left(\Omega, \mathcal{F}, R^{n}\right)$ such that $g(t) \in G(t)$ for all $t \geq 0$. Obviously, continuity with respect to $\tau_{\rightarrow}$ means the right-hand continuity of $g$. We can then define the stochastic process $\left(f_{t}\right)_{t>0}$ by $f_{t}(\omega)=g(t)(\omega)$. Clearly, a selection $\left(f_{t}\right)$ is $\mathcal{F}_{t^{-}}$ adapted. Since $E\left(\left|f_{t}-f_{s}\right|\right)=E(|g(t)-g(s)|) \rightarrow 0$ as $t \downarrow s$, then by the Chebyshev inequality, $P\left(\left|f_{t}-f_{s}\right|>\epsilon\right) \rightarrow 0$ as $t \rightarrow s$. Thus, $\left(f_{t}, \mathcal{F}_{t}\right)$ is stochastically right-hand continuous.

For the proof of the next selection theorem, we will need also the following consequence of Levy's martingale convergence theorem.

Proposition 1: $\mathscr{F}_{t}=\mathscr{F}_{t}$ - if and only if the function $s \mapsto E\left(\varphi \mid \mathscr{F}_{s}\right)$ is P-almost everywhere left-hand continuous at $t$ for each $\mathcal{F}_{t}$-measurable $\varphi$ such that $E(|\varphi|)<$ $+\infty$. Analogously, $\mathcal{F}_{t}=\mathcal{F}_{t+}$ if and only if the function $s \mapsto E\left(\varphi \mid \mathcal{F}_{s}\right)$ is P-almost

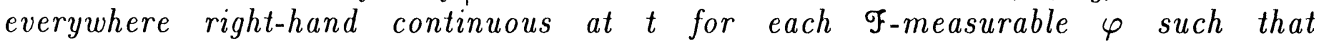
$E(|\varphi|)<+\infty$.

Proof: If $\mathcal{F}_{t}=\mathcal{F}_{t-}$, then by Levy's theorem (see Liptser and Shiraev [6, p. 24]) 
we have that $E\left(\varphi \mid \mathcal{F}_{s_{n}}\right) \rightarrow E\left(\varphi \mid \mathscr{F}_{t}\right)$ whenever $s_{n} \uparrow t$. Conversely, observe that for $A \in \mathcal{F}_{t}, E\left(\chi_{A} \mid \mathscr{F}_{s_{n}}\right) \rightarrow E\left(\chi_{A} \mid \mathcal{F}_{t_{-}}\right)$by Levy's theorem whenever $s_{n} \uparrow t$. On the other hand, by assumption $E\left(\chi_{A} \mid \mathscr{F}_{s_{n}}\right) \rightarrow E\left(\chi_{A} \mid \mathscr{F}_{t}\right)=\chi_{A}$, thus $\chi_{A}=E\left(\chi_{A} \mid \mathscr{F}_{t-}\right) P$ almost everywhere. Therefore, ${ }^{n}$ for $B=\left(E_{\chi_{A}} \mid \mathcal{F}_{t-}\right)^{-1}(1) \in F_{t-}, P((A \backslash B) \cup$ $(B \backslash A))=0$. Since all $P$-null sets are in $\mathcal{F}_{t-}$, we conclude that $A \in \mathcal{F}_{t-}$. The analogous statement regarding $\mathcal{F}_{t}=\mathcal{F}_{t+}$ can be verified in the same way.

Theorem 3: Let $\mathcal{F}_{t}=\mathcal{F}_{t}$ - for each $t>0$. Assume that a set-valued stochastic process $\left(F_{t}, \mathcal{F}_{t}\right)$ has closed values and for every $z \in R^{n}, s \geq 0, A \in \mathcal{F}_{s}$, the real-valued function $t \mapsto E\left(\chi_{A} d\left(z, F_{t}\right)\right)$ is usc at $s$. Assume also that $P$ is nonatomic or $\left(F_{t}, \mathscr{F}_{t}\right)$ has convex values. Then $\left(F_{t}, \mathcal{F}_{t}\right)$ has an $L^{1}$-continuous selection $\left(f_{t}, \mathcal{F}_{t}\right)$.

Proof: We consider $[0,+\infty)$ with the usual topology and will show that $G$ (defined in the proof of Theorem 2) is lower semicontinuous. The right-hand lower semicontinuity can be proved exactly in the same way as in Theorem 2, so it suffices to show that for fixed $s>0, \varphi \in G(s)$, we have $d(\varphi, G(t)) \rightarrow 0$ as $t \uparrow s$. But for $t<s$, we have

$$
\begin{gathered}
d(\varphi, G(t)) \leq E\left(\left|\varphi-E\left(\varphi \mid \mathcal{F}_{t}\right)\right|\right)+d\left(E\left(\varphi \mid \mathcal{F}_{t}\right), G(t)\right) \\
\left.=E\left(\left|\varphi-E\left(\varphi \mid \mathcal{F}_{t}\right)\right|\right)+E\left(d\left(E\left(\varphi \mid \mathcal{F}_{t}\right), F_{t}\right)\right)\right) \\
\leq E\left(\left|\varphi-E\left(\varphi \mid \mathcal{F}_{t}\right)\right|\right)+E\left(\left|E\left(\varphi \mid \mathcal{F}_{t}\right)-\varphi\right|\right)+E\left(d\left(\varphi, F_{t}\right)\right) .
\end{gathered}
$$

By Proposition 1 we have $E\left(\left|\varphi-E\left(\varphi \mid \mathcal{F}_{t}\right)\right|\right) \rightarrow 0$ as $t \uparrow s$, and by Lemma 2 we have $E\left(d\left(\varphi, F_{t}\right)\right) \rightarrow 0$ as $t \uparrow s$. Therefore $G$ is a lower semicontinuous set-valued mapping with closed values. Suppose now that $P$ is nonatomic. Clearly, the sets $G(t)$ are decomposable (i.e., $\varphi \chi_{A}+\psi \chi_{\Omega \backslash A} \in G(t)$ whenever $\varphi, \psi \in G(t)$ and $A \in \mathscr{F}_{t}$ ). We can apply the Antosiewicz-Cellina continuous selection theorem (see Bressan and Colombo [1, Theorem 3]) to $G$, and get a continuous mapping $g:[0,+\infty) \rightarrow$ $L^{1}\left(\Omega, \mathscr{F}, R^{n}\right)$ such that $g(t) \in G(t)$ for all $t \geq 0$. If $\left(F_{t}, \mathscr{F}_{t}\right)$ has convex values, as in the proof of Theorem 2, we get a continuous selection $g$ applying Michael's theorem. Thus, the stochastic process $\left(f_{t}\right)$ defined by $f_{t}(\omega)=g(t)(\omega)$ has desired properties.

If we assure the continuity of the conditional expectation operator $t \mapsto E\left(\varphi \mid \mathcal{F}_{t}\right)$, then we can extend Hess' result [3, Theorem 3.2] on the martingale selection of discrete time set-valued martingale and obtain a continuous martingale selection result. A set-valued process $\left(F_{t}, \mathscr{F}_{t}\right)$ is a set-valued martingale if

$$
\begin{aligned}
& \left\{\varphi \in L^{1}(\Omega, \mathcal{F}, \mathscr{P}): \varphi \text { is } \mathscr{F}_{s} \text {-measurable selection of } F_{s}\right\} \\
& =\operatorname{cl}\left\{E\left(\varphi \mid \mathscr{F}_{s}\right): \varphi \text { is } \mathscr{F}_{t} \text {-measurable selection of } \mathscr{F}_{t}\right\}
\end{aligned}
$$

for any $0 \leq s \leq t$, (see Hiai and Umegaki [4], Hess [3]). We propose the following continuous time version of Hess' theorem.

Proposition 2: Let $\left(F_{t}, \mathcal{F}_{t}\right)$ be a set-valued martingale. If for every $t \geq 0$ we have $\mathcal{F}_{t}=\mathcal{F}_{t-}$, then $\left(F_{t}, \mathcal{F}_{t}\right)$ admits a martingale selection $\left(f_{t}, \mathcal{F}_{t}\right)$ with $P$-almost all paths left-hand continuous. If for every $t \geq 0$ we have $\mathcal{F}_{t}=\mathcal{F}_{t+}$, then $\left(F_{t}, \mathcal{F}_{t}\right)$ admits a martingale selection $\left(f_{t}, \mathcal{F}_{t}\right)$ with $P$-almost all paths right-hand continuous.

Proof: Consider the discrete time set-valued martingale $\left(F_{n}\right)_{n=0, \ldots}$ obtained 
from $\left(F_{t}, \mathcal{F}_{t}\right)$ by taking $t=0,1, \ldots$ By the Hess result, $\left(F_{n}\right)$ has a martingale selection $\left(f_{n}\right)$ (i.e., there exists a sequence of $\mathscr{F}_{n}$-measurable mappings $f_{n}: \Omega \rightarrow R^{n}$ such that $f_{n}$ is a selection of $F_{n}$ and $f_{n}=E\left(f_{n+1} \mid F_{n}\right)$ for $\left.n=0,1, \ldots\right)$. For $t \in$ $[0,+\infty) \backslash\{0,1,2, \ldots\}$ we define $f_{t}: \Omega \rightarrow R^{n}$ by $f_{t}=E\left(f_{n} \mid \mathscr{F}_{t}\right)$ where $n-1<t<n$. Clearly, $\left(f_{t}\right)$ is a martingale selection of $F$. By Proposition $1,\left(f_{t}\right)$ has $P$-almost all paths left-hand (respectively, right-hand) continuous.

\section{References}

[1] Bressan, A. and Colombo, G., Extensions and selections of maps with decomposable values, Studia Math 90 (1988), 69-85.

[2] Engelking, R., General Topology, Monografie Matematyczne, PWN Warszawa, Poland 1976.

[3] Hess, C., On multivalued martingales whose values may be unbounded: Martingale selectors and Mosco convergence, J. Multivar. Anal. 39 (1991), 175-201.

[4] Hiai, F. and Umegaki, H., Integrals, conditional expectations, and martingales of multivalued functions, J. Multivar. Anal. 7 (1977), 149-182.

[5] Himmelberg, C.J., Measurable relations, Fund. Math. 87 (1975), 53-72.

[6] Liptser, R.Sh. and Shiraev, A.N., Stochastics of Random Processes, SpringerVerlag, Berlin 1976.

[7] Michael, E., Continuous selections I, Ann. of Math. 63 (1956), 361-382.

[8] Rybiński, L.E., Random fixed points and viable random solutions of functionaldifferential inclusions, J. Math. Anal. Appl. 142 (1989), 53-61.

[9] Salinetti, G. and Wets, R.J.-B., On the convergence in distribution of measurable multifunctions (random sets), normal integrands, stochastic processes and stochastic infima, Math. Oper. Res. 11 (1986), 385-419.

[10] Wijsman, R.A., Convergence of sequences of convex sets, cones and functions, Bull. Amer. Math. Soc. 70 (1964), 186-188. 


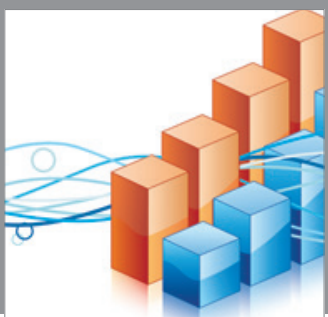

Advances in

Operations Research

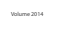

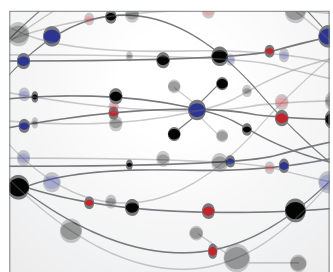

\section{The Scientific} World Journal
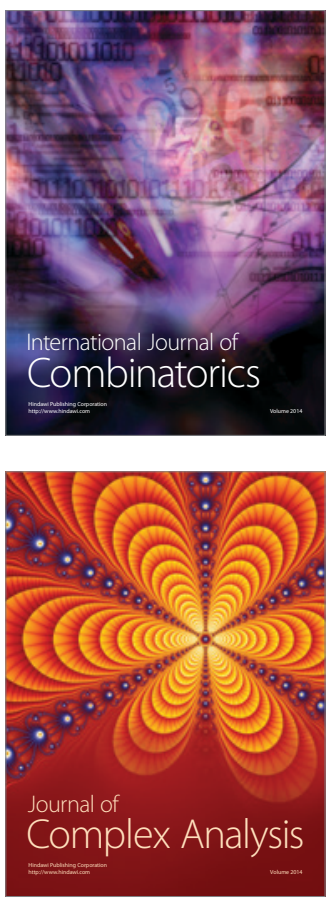

International Journal of

Mathematics and

Mathematical

Sciences
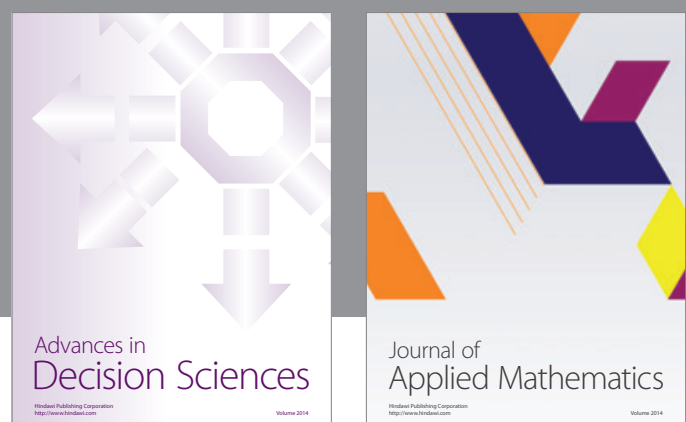

Journal of

Applied Mathematics
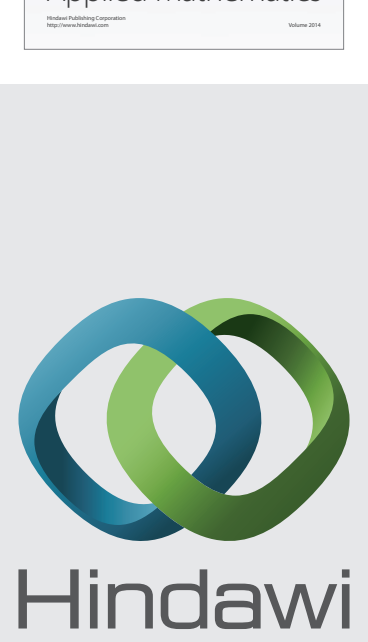

Submit your manuscripts at http://www.hindawi.com
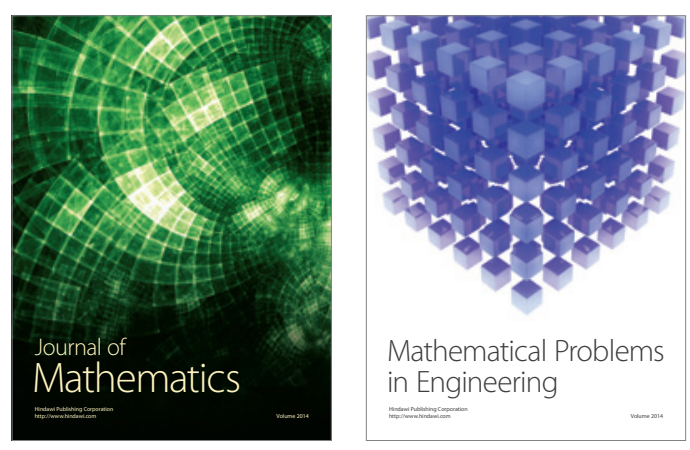

Mathematical Problems in Engineering
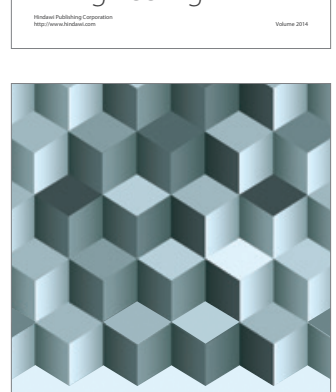

Journal of

Function Spaces
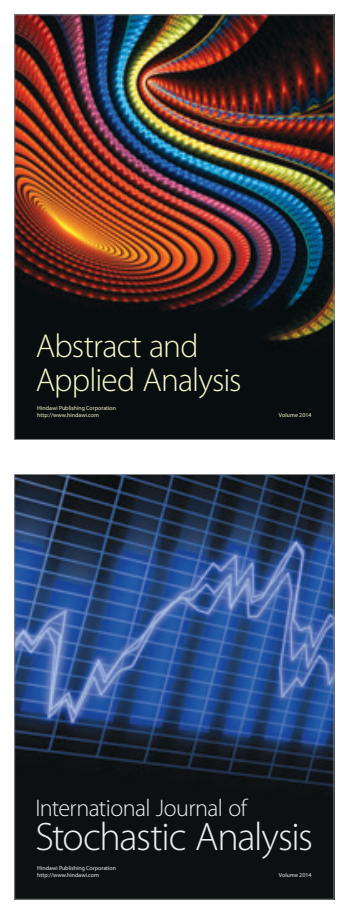

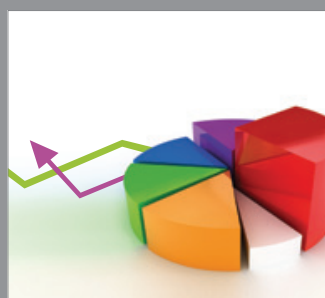

ournal of

Probability and Statistics

Promensencen
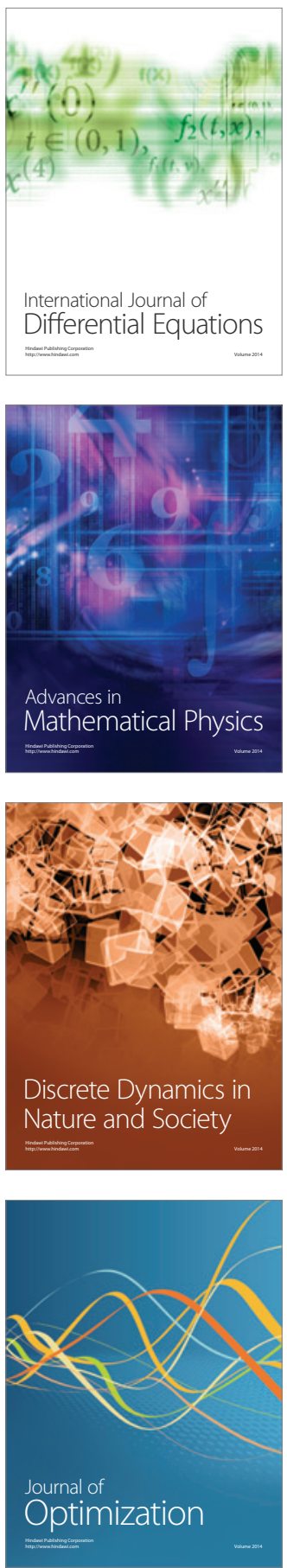